EPJ Web of Conferences 97,00023 (2015)

DOI: $10.1051 /$ epjconf/ 20159700023

(C) Owned by the authors, published by EDP Sciences, 2015

\title{
The two $\Lambda(1405)$ poles from photoproduction data
}

\author{
Eulogio Oset ${ }^{1, a}$ and Luis Roca ${ }^{2, b}$ \\ ${ }^{1}$ Departamento de Física Teórica and IFIC, Centro Mixto Universidad de Valencia-CSIC, Institutos de \\ Investigación de Paterna, Aptdo. 22085, 46071 Valencia, Spain \\ ${ }^{2}$ Departamento de Física, Universidad de Murcia, E-30100 Murcia, Spain
}

\begin{abstract}
We present results extracting the position of the two $\Lambda(1405)$ poles from experimental photoproduction data measured recently at different energies in the $\gamma p \rightarrow K^{+} \pi^{0} \Sigma^{0}$ and $\gamma p \rightarrow K^{+} \pi^{ \pm} \Sigma^{\mp}$ reactions at Jefferson Lab. The analysis is done by means of a chiral dynamics motivated potential but with free parameters, which guarantees analyticity and unitarity in the solutions. With the best result we find the two $\Lambda(1405)$ poles at about $1352-48 i \mathrm{MeV}$ and $1419-29 i \mathrm{MeV}$.
\end{abstract}

\section{Introduction}

The issue of the nature of the $\Lambda(1405)$ has captured great attention through the years. Very early it was already postulated that it could be a resonance made from the interaction of the coupled channels $\bar{K} N$ and $\pi \Sigma$ [1]. A big step forward was made possible with the use of chiral dynamics in its unitarized form, the so called chiral unitary approach, which has brought a new perspective to the problem and has shown the importance of coupled channels and unitarity [2-11]. One of the novel aspects of these works has been the finding of two poles, and thus two states, rather than one, associated to the experimental peaks of the $\Lambda(1405)$ resonance. Due to the existence of these two poles, the peak observed in experiments should be different in different reactions, as has been the case in the reactions studied so far [12-19]. The early experiments gave a peak around $1405 \mathrm{MeV}$, which served to give the nominal mass to the resonance. In view of this, new reactions were devised that would show the peak around $1420 \mathrm{MeV}$, close to the second pole found in the chiral unitary approach works. A first suggestion was made in [20], where the radiative production of the $\Lambda(1405)$ resonance in $K^{-}$collisions on protons was proposed, and a narrow peak around $1420 \mathrm{MeV}$ was predicted. Awaiting for this reaction to be done, an equivalent reaction, the $K^{-} p \rightarrow \pi^{0} \pi^{0} \Sigma^{0}$ [15], was measured and a peak was indeed seen around $1420 \mathrm{MeV}$ and narrower than the one observed in $[12,13]$. A theoretical description of this reaction in terms of the chiral unitary approach of [3] was provided in [21]. Further support for the two pole picture came from [22] where the $K^{-} d \rightarrow n \pi \Sigma$ reaction was measured and a neat peak was observed around $1420 \mathrm{MeV}$. The reaction was studied theoretically in [23] and it was found that the mechanism of scattering

\footnotetext{
a e-mail: oset@ific.uv.es

be-mail: luisroca@um.es
}

This is an Open Access article distributed under the terms of the Creative Commons Attribution License 4.0, which permits unrestricted use, distribution, and reproduction in any medium, provided the original work is properly cited. 
of the kaon with a neutron, losing some energy, followed by rescattering of the kaon with the proton to produce the $\Lambda(1405)$, provided the right strength and shape observed in experiment.

The surprising thing is that the theoretical approaches dealing with the $\bar{K} N$ interaction and predicting the properties of the $\Lambda(1405)$ have paid little or null attention to the reactions where the resonance is produced, although in Ref. [9] some attention is given to $\Lambda(1405)$ production. One of the exceptions to this rule is the model constructed for photoproduction of the $\Lambda(1405)$, done in [24] before the experiment was performed, which predicted the basic features and strength of the reaction. Similarly, the $\pi^{-} p \rightarrow K^{0} \pi \Sigma$ reaction of [12] was studied theoretically in [25], the $p p \rightarrow p K^{+} \pi \Sigma$ reaction of [18] in [26], the $K^{-} p \rightarrow \pi^{0} \pi^{0} \Sigma^{0}$ of [15] in [21] and the $K^{-} d \rightarrow n \pi \Sigma$ reaction of [22] in [23]. The chiral unitary approach with the potential from the lowest order chiral Lagrangians was used in all these studies. Meanwhile more refined models have been developed [8-11] that contain the next to leading order terms in the potential. It is, however, interesting to observe that the results of [3] with the lowest order potential provide all the observables on cross sections and threshold ratios within the error bands provided by the more refined theoretical potential of [10].

In the present talk we present results from a fit to the photoproduction of $\Lambda(1405)$ at different energies of the CLAS collaboration at Jefferson Lab [16, 17]. For this purpose we have taken the $\pi \Sigma$ and $\bar{K} N$ states in isospin $\mathrm{I}=0$ and $\mathrm{I}=1$ and solved the coupled channels Bethe Salpeter equations in terms of a potential suggested by chiral dynamics but with free parameters. We show that the fit determines the potential with a precision that allows one to conclude that there are two poles, one around $1350 \mathrm{MeV}$ and wide and another one around $1420 \mathrm{MeV}$ and narrow, like in most chiral unitary approaches fitting scattering data.

\section{Unitarized meson-baryon amplitude}

In the chiral unitary approach one starts from the lowest order chiral Lagrangian for the interaction of the octet of Goldstone bosons with the octet of the low lying $1 / 2^{+}$baryons [27], which provides the following tree level transition amplitudes in $s$-wave [28]:

$$
V_{i j}^{I}(\sqrt{s})=-C_{i j}^{I} \frac{1}{4 f^{2}}\left(2 \sqrt{s}-M_{i}-M_{j}\right)\left(\frac{M_{i}+E_{i}}{2 M_{i}}\right)^{1 / 2}\left(\frac{M_{j}+E_{j}}{2 M_{j}}\right)^{1 / 2},
$$

where the superscript $I$ stands for the isospin, $\sqrt{s}$ the center of mass energy, $f$ the averaged meson decay constant $f=1.123 f_{\pi}[28]$ with $f_{\pi}=92.4 \mathrm{MeV}, E_{i}\left(M_{i}\right)$ the energies (masses) of the baryons of the $i$-th channel. The $C_{i j}^{0}$ coefficients, for isospin $I=0$, are given by

$$
C_{i j}^{0}=\left(\begin{array}{cc}
3 & -\sqrt{\frac{3}{2}} \\
-\sqrt{\frac{3}{2}} & 4
\end{array}\right)
$$

The $i$ and $j$ subscripts represent the channels $\bar{K} N$ and $\pi \Sigma$ in isospin-basis. Note that we do not consider the other possible channels in $I=0, \eta \Lambda$ and $K \Xi$, for the sake of simplicity of the approach and because for the energies that we will consider in this work the effect of those channels can be effectively reabsorbed in the subtraction constants. The coefficients for isospin $I=1$ are

$$
C_{i j}^{1}=\left(\begin{array}{ccc}
3 & -1 & -\sqrt{\frac{3}{2}} \\
-1 & 2 & 0 \\
-\sqrt{\frac{3}{2}} & 0 & 0
\end{array}\right) .
$$


where the order of the channels are $\bar{K} N, \pi \Sigma$ and $\pi \Lambda$. We also neglect here the $\eta \Sigma$ and $K \Xi$ states into the coupled channels equations since their thresholds are also very far from the energy region of interest in the present work.

The chiral unitary approach is based on the implementation of unitarity of the scattering amplitude in coupled channels and the exploitation of its analytic properties. This is accomplished, among other methods, using the Bethe-Salpeter equations in coupled-channel and the scattering amplitude $T_{i j}$ is given by the matrix equation

$$
T=[1-V G]^{-1} V
$$

where $V_{i j}$ is the interaction kernel of Eq. (1) and the function $G_{i}$, or unitary bubble, is the meson-baryon loop function which is logarithmically divergent, and therefore must be regularized, usually carried out either with a three momentum cutoff or with dimensional regularization in terms of a subtraction constant $a_{i}$. In ref. $[6,28]$ the values $a_{K N}=-1.84$, $a_{\pi \Sigma}=-2$ where used for the $I=0$ channels. In the present case, since we do not consider the $\eta \Lambda$ and $K \Xi$ channels, these subtraction constants may differ slightly but we will allow to vary these constants in the fit below. For the $I=1$ channels, in ref.[29] the same value for $a_{K N}, a_{\pi \Sigma}$ as in the $I=0$ case was used and $a_{\pi \Lambda}=-1.83$ for the new channel in the $I=1$.

It is worth mentioning that the resonant shapes of the amplitudes obtained are far from looking like Breit-Wigner shapes. Therefore fits to experimental data assuming Breit-Wigner resonant shapes are not suitable for this resonance and a model like the present one, implementing unitarity in coupled channels, is more suited to reproduce experimental data.

\section{Fit to photoproduction data}

In terms of isospin states we have the following decomposition of the final $\pi \Sigma$ states,

$$
\begin{aligned}
\left|\pi^{0} \Sigma^{0}\right\rangle & =\sqrt{\frac{2}{3}}|20\rangle-\frac{1}{\sqrt{3}}|00\rangle, \\
\left|\pi^{+} \Sigma^{-}\right\rangle & =-\frac{1}{\sqrt{6}}|20\rangle-\frac{1}{\sqrt{2}}|10\rangle-\frac{1}{\sqrt{3}}|00\rangle, \\
\left|\pi^{-} \Sigma^{+}\right\rangle & =-\frac{1}{\sqrt{6}}|20\rangle+\frac{1}{\sqrt{2}}|10\rangle-\frac{1}{\sqrt{3}}|00\rangle,
\end{aligned}
$$

Since the $\Lambda(1405)$ is dynamically generated from the final state interaction of the mesonbaryon pair produced, and we also seek for a possible generated resonance from the mesonbaryon scattering in $I=1$, the most general mechanisms for the photoproduction reaction are those depicted in fig. 1 . The photoproduction can proceed by the production of either a $\pi \Sigma$ or $\bar{K} N$ pair for $I=0$ and $I=1$ and also by $\pi \Lambda$ for the $I=1$ case. This initial production is represented by the thick circle in fig. 1. The initial meson-baryon pair then rescatters to produce the final $\pi \Sigma$, accounted for by the unitarized scattering amplitude explained in the previous section. 


\section{EPJ Web of Conferences}
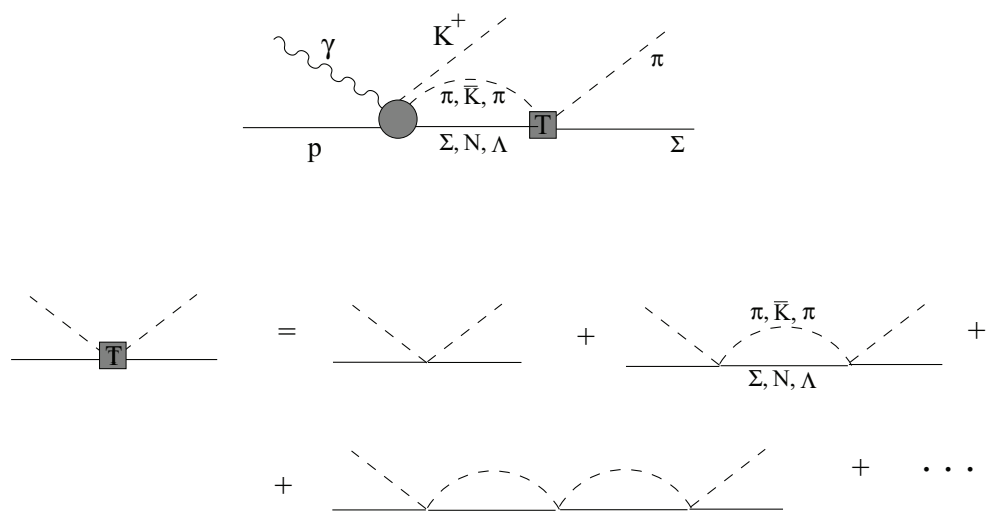

Figure 1. General mechanisms for the photoproduction amplitudes

Based on fig. 1, the photoproduction amplitudes can be written as

$$
\begin{aligned}
t_{\gamma p \rightarrow K^{+} \pi^{0} \Sigma^{0}}(W) & =b_{0}(W) G_{\pi \Sigma}^{I=0} T_{\pi \Sigma, \pi \Sigma}^{I=0}+c_{0}(W) G_{\bar{K} N}^{I=0} T_{\bar{K} N, \pi \Sigma}^{I=0} \\
t_{\gamma p \rightarrow K^{+} \pi^{ \pm} \Sigma^{\mp}}(W) & =b_{0}(W) G_{\pi \Sigma}^{I=0} T_{\pi \Sigma, \pi \Sigma}^{I=0}+c_{0}(W) G_{\bar{K} N}^{I=0} T_{\bar{K} N, \pi \Sigma}^{I=0} \\
& \pm \sqrt{\frac{3}{2}}\left(b_{1}(W) G_{\pi \Sigma}^{I=1} T_{\pi \Sigma, \pi \Sigma}^{I=1}+c_{1}(W) G_{\bar{K} N}^{I=1} T_{\bar{K} N, \pi \Sigma}^{I=1}\right. \\
& \left.+d_{1}(W) G_{\pi \Lambda}^{I=1} T_{\pi \Lambda, \pi \Sigma}^{I=1}\right)
\end{aligned}
$$

with $W$ the energy of the $\gamma p$ interaction. The subindex in the $b, c$ and $d$ coefficients stand for the isospin. Note that the only difference between the $\gamma p \rightarrow K^{+} \pi^{+} \Sigma^{-}$and the $\gamma p \rightarrow$ $K^{+} \pi^{-} \Sigma^{+}$amplitudes is the sign of the $I=1$ contributions. The coefficients $b, c$ and $d$ may in general depend on $W$ and hence we consider 9 sets of them in order to account for the 9 different energies $W$ provided by the experimental result of CLAS [17]. On the other hand the relative weight between the different $G T$ addends may be complex in general, therefore we allow the $b_{1}, c_{0}, c_{1}$ and $d_{1}$ to be complex and keep $b_{0}$ real since a global phase in the total amplitude is irrelevant. We will refer the $b, c$ and $d$ coefficients by initial production (IP) parameters in the following.

In $[30,31]$ it is shown that already good results are obtained fitting only the IP parameters and keeping the chiral parameters like in the original works. But since we want to extract information from photoproduction, we can try to get results with better $\chi^{2} /$ dof by allowing the basic chiral unitary model to vary slightly. In this way we could obtain a fine tuning of the chiral unitary model and then of the position of the $\Lambda(1405)$ poles and try to see if some $I=1$ resonance shows up. In order to do this we multiply each coefficient of the potentials of the unitary amplitudes, Eqs. (2) and (3), by one real parameter $\alpha_{i}$ and hence the new coefficient matrices that we consider now are given by

$$
C_{i j}^{0}=\left(\begin{array}{cc}
3 \alpha_{11}^{0} & -\sqrt{\frac{3}{2}} \alpha_{12}^{0} \\
-\sqrt{\frac{3}{2}} \alpha_{12}^{0} & 4 \alpha_{22}^{0}
\end{array}\right)
$$


for isospin $I=0$ and

$$
C_{i j}^{1}=\left(\begin{array}{ccc}
3 \alpha_{11}^{1} & -\alpha_{12}^{1} & -\sqrt{\frac{3}{2}} \alpha_{13}^{1} \\
-\alpha_{12}^{1} & 2 \alpha_{22}^{1} & 0 \\
-\sqrt{\frac{3}{2}} \alpha_{13}^{1} & 0 & 0
\end{array}\right)
$$

for isospin $I=1$.

Furthermore we also allow to vary the subtraction constants from the regularization of the loop functions by multiplying each of them by a free parameter, $\beta_{i}: a_{K N} \rightarrow \beta_{1} a_{K N}$, $a_{\pi \Sigma} \rightarrow \beta_{2} a_{\pi \Sigma}$ and $a_{\pi \Lambda} \rightarrow \beta_{3} a_{\pi \Lambda}$. We will refer to the $\alpha$ and $\beta$ parameters by potential parameters in the following (even though the $\beta$ coefficients do not affect the potential, but we do this just to ease the nomenclature). Therefore, the chiral unitary amplitudes depend on 10 free parameters to be fitted, $\alpha_{i}, \beta_{i}$, but only 5 of them affect the $I=0$ amplitude and 7 the $I=1$. With the potential obtained from the fit we shall search for the positions of the two $\Lambda(1405)$ poles and look for a possible $I=1$ resonance in the range of energy considered.

In order to get physically meaningful results, we implement the following strategy: As mentioned above, the fit to the data fixing the potential parameters to 1 , is already reasonably fair, and the potential is consistent with data of scattering [3], hence a good physical global fit should not be very far from having values of $\alpha_{i} \sim 1, \beta_{i} \sim 1$. Therefore, in a first step, we start from the fit which was obtained fixing the potential parameters to $1\left(\alpha_{i}=1, \beta_{i}=1\right)$, but fixing now the IP parameters and allowing only the potential parameters to change. In a next step, we fix the new potential parameters obtained in the previous step and fit again the IP parameters. We iterate the process alternating between fitting the IP or fitting the potential parameters until we get a convergence of the value of the $\chi^{2}$. In this way we obtain a minimum of the $\chi^{2}$ with potential parameters not very different from 1 which are then physically meaningful.

After this iterative procedure we get the result shown in fig. 2, which has $\chi^{2} /$ dof $=2.1$. The bands account for the uncertainties of the fit at one standard deviation confidence level. The potential parameters obtained are shown in table 1 .

Table 1. Parameters of the unitarized amplitudes

\begin{tabular}{cccccccccc}
\hline \hline$\alpha_{11}^{0}$ & $\alpha_{12}^{0}$ & $\alpha_{22}^{0}$ & $\alpha_{11}^{1}$ & $\alpha_{12}^{1}$ & $\alpha_{13}^{1}$ & $\alpha_{22}^{1}$ & $\beta_{1}$ & $\beta_{2}$ & $\beta_{3}$ \\
\hline 1.037 & 1.466 & 1.668 & 0.85 & 0.93 & 1.056 & 0.77 & 1.187 & 0.722 & 1.119 \\
\hline \hline
\end{tabular}

It is important to note that the parameters obtained are not very different from one.

In table 2 we show the results obtained for the pole positions in the complex energy $(\sqrt{s})$ plane in unphysical Riemann sheets of the scattering amplitudes.

Table 2. Pole positions (in MeV) in the complex energy plane of the scattering amplitudes and modulus of the couplings to the different channels.

\begin{tabular}{|c|c|c|c|}
\hline \hline & \multicolumn{2}{|c|}{$I=0$} & $I=1$ \\
\hline poles & $1352-48 i$ & $1419-29 i$ & - \\
\hline$\left|g_{\bar{K} N}\right|$ & 2.71 & 3.06 & - \\
\hline$\left|g_{\pi \Sigma}\right|$ & 2.96 & 1.96 & - \\
\hline \hline
\end{tabular}




\section{EPJ Web of Conferences}
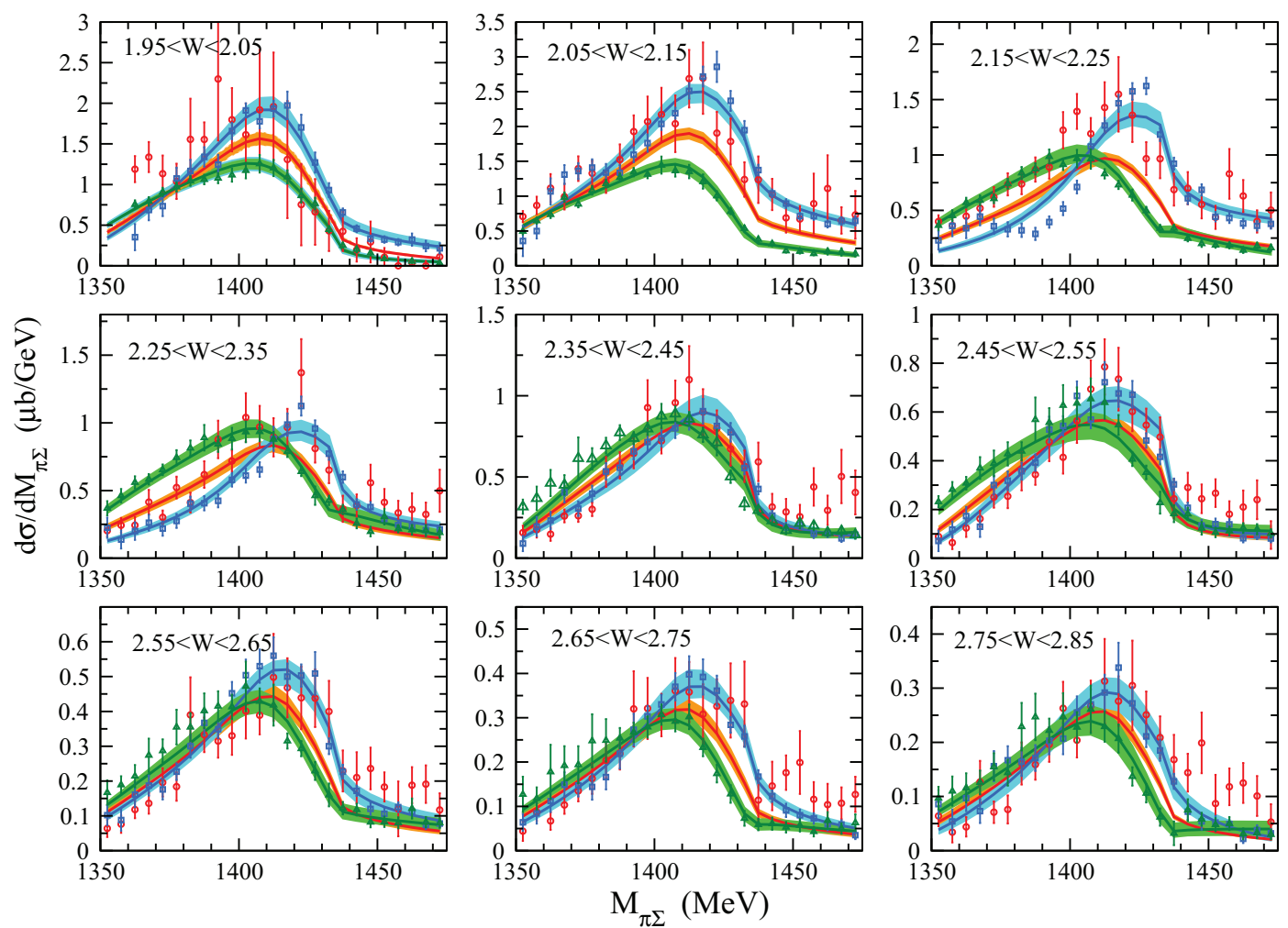

Figure 2. (Color online) solution from the fit procedure described in the text

In [31] we also discuss that no pole is found for $I=1$ in the ordinary Riemann sheet, but there is a pole located at $1522-14 i \mathrm{MeV}$ in another unphysical Riemann sheet which does not produce a Breit-Wigner shape in the real axis but has instead the appearance of a pronounced cusp at the $\bar{K} N$ threshold.

In order to make further checks that the fit obtained is physically acceptable, we calculate now the cross section for $K^{-} p \rightarrow M B$ for the meson-baryon final channels $K^{-} p, K^{0} n, \pi^{+} \Sigma^{-}$, $\pi^{-} \Sigma^{+}, \pi^{0} \Sigma^{0}$ and $\pi^{0} \Lambda$. The results are shown in [31] in comparison to experimental data and the agreement is quite good.

Another experimental data usually considered in other theoretical works [32-34] regarding the $\Lambda(1405)$ resonance are the energy shift and width of the kaonic hydrogen in the $1 s$ state from the SIDDHARTA experiment at DAFNE [35], which are reported to be $\Delta E-i \Gamma / 2=$ $(283 \pm 42)-i(271 \pm 55) \mathrm{eV}$. This value is related to the $K^{-} p$ scattering length and therefore to the $K^{-} p \rightarrow K^{-} p$ amplitude at threshold. (For explicit mathematical expressions see refs. [32-34]). With the values of the parameters in table 1 we obtain $\Delta E-i \Gamma / 2=(194 \pm$ $4)-i(301 \pm 9) \mathrm{eV}$, which compares reasonably well to the experimental SIDDHARTA data.

On the other hand, in a different fit to the CLAS data made by some members of that collaboration [36], two different kind of fits were performed: one only to the $\pi^{0} \Sigma^{0}$ data, to which only the $I=0$ channel contributes and one to all the photoproduction data. The 
amplitudes in that fit are parametrized as (Eq.(5) of [36])

$$
t_{I}(m)=C_{I}(W) e^{i \Delta \phi_{I}} B_{I}(m)
$$

where $C_{I}(W)$ is a weight factor, $\Delta \phi_{I}$ a phase and $B_{I}(m)$ a Breit-Wigner function. As one can see, the weight is allowed to depend on the photon energy, $W$, but not its phase. But even more restrictive is the fact that the shapes of the resonances, $B_{I}(m)$, are Breit-Wigner shapes and chosen independent of the photon energy. This neglects the possibility that the amplitudes $\gamma p \rightarrow K^{+} \pi \Sigma$ are superpositions of the amplitudes corresponding to the different poles with relative weights that depend on the photon energy. Furthermore, as seen in the plots of the amplitudes throughout the present work, the resonant amplitudes are far from being Breit-Wigner like.

For comparison we quote here the results of [36] when a fit to the data is done including two Breit-Wigners for $I=0$ and one for $I=1$, where the authors get $M=1338, \Gamma=44$, for one of the $I=0$ resonances and $M=1384, \Gamma=76$, for the other one and $M=1357, \Gamma=54$, for the $I=1$. This fit should be compared to ours in the present paper, (see table 2). The arguments exposed above can explain the difference in the results of our fit and CLAS. It is worth noting that a recent paper [37] including higher order terms in the potential and fitting scattering and photoproduction data obtain results remarkably similar to those presented here .

\section{Summary}

We have made a fit to the $\gamma p \rightarrow K^{+} \pi \Sigma$ experimental data by using a chiral unitary approach but with free parameters for the meson baryon scattering potential. We show that the data demand two states in $I=0, \Lambda(1405)$ states and there is a pronounced cusp structure $I=1$.

Fair results are then obtained for the cross sections of the $K^{-} p \rightarrow M B$ for the mesonbaryon final channels $K^{-} p, K^{0} n, \pi^{+} \Sigma^{-}, \pi^{-} \Sigma^{+}, \pi^{0} \Sigma^{0}$ and $\pi^{0} \Lambda$ and for experimental data on kaonic hydrogen. The new photoproduction data have been essential to set on firm grounds the previous findings of most of the studies based on the chiral unitary approach.

\section{Acknowledgments}

This work is partly supported by the Spanish Ministerio de Economia y Competitividad and European FEDER funds under the contract number FIS2011-28853-C02-01, and the Generalitat Valenciana in the program Prometeo II- 2014/068. We acknowledge the support of the European Community-Research Infrastructure Integrating Activity Study of Strongly Interacting Matter (acronym HadronPhysics3, Grant Agreement n. 283286) under the Seventh Framework Programme of EU.

\section{References}

[1] R. H. Dalitz, S. F. Tuan, Annals Phys. 10, 307-351 (1960).

[2] N. Kaiser, P. B. Siegel and W. Weise, Nucl. Phys. A 594, 325 (1995).

[3] E. Oset and A. Ramos, Nucl. Phys. A 635, 99 (1998).

[4] J. A. Oller and U. G. Meissner, Phys. Lett. B 500, 263 (2001).

[5] T. Hyodo, S. I. Nam, D. Jido and A. Hosaka, Phys. Rev. C 68, 018201 (2003). 


\section{EPJ Web of Conferences}

[6] D. Jido, J. A. Oller, E. Oset, A. Ramos, U. G. Meissner, Nucl. Phys. A725, 181-200 (2003).

[7] C. Garcia-Recio, J. Nieves, E. Ruiz Arriola and M. J. Vicente Vacas, Phys. Rev. D 67, 076009 (2003).

[8] B. Borasoy, R. Nissler and W. Weise, Eur. Phys. J. A 25, 79 (2005).

[9] J. A. Oller, Eur. Phys. J. A 28, 63 (2006).

[10] B. Borasoy, U. G. Meissner and R. Nissler, Phys. Rev. C 74, 055201 (2006).

[11] Y. Ikeda, T. Hyodo and W. Weise, Nucl. Phys. A 881, 98 (2012).

[12] D. W. Thomas, A. Engler, H. E. Fisk and R. W. Kraemer, Nucl. Phys. B 56, 15 (1973).

[13] R. J. Hemingway, Nucl. Phys. B 253, 742 (1985).

[14] M. Niiyama, H. Fujimura, D. S. Ahn, J. K. Ahn, S. Ajimura, H. C. Bhang, T. H. Chang and W. C. Chang et al., Phys. Rev. C 78, 035202 (2008).

[15] S. Prakhov et al. [Crystall Ball Collaboration], Phys. Rev. C 70, 034605 (2004).

[16] K. Moriya et al. [CLAS Collaboration], AIP Conf. Proc. 1441, 296 (2012).

[17] K. Moriya et al. [CLAS Collaboration], arXiv:1301.5000 [nucl-ex].

[18] I. Zychor, M. Buscher, M. Hartmann, A. Kacharava, I. Keshelashvili, A. Khoukaz, V. Kleber and V. Koptev et al., Phys. Lett. B 660, 167 (2008).

[19] G. Agakishiev, A. Balanda, D. Belver, A. V. Belyaev, J. C. Berger-Chen, A. Blanco, M. Bohmer and J. L. Boyard et al., arXiv:1208.0205 [nucl-ex].

[20] J. C. Nacher, E. Oset, H. Toki and A. Ramos, Phys. Lett. B 461, 299 (1999).

[21] V. K. Magas, E. Oset and A. Ramos, Phys. Rev. Lett. 95, 052301 (2005).

[22] O. Braun, H. J. Grimm, V. Hepp, H. Strobele, C. Thol, T. J. Thouw, D. Capps and F. Gandini et al., Nucl. Phys. B 129, 1 (1977).

[23] D. Jido, E. Oset and T. Sekihara, Eur. Phys. J. A 42, 257 (2009).

[24] J. C. Nacher, E. Oset, H. Toki and A. Ramos, Phys. Lett. B 455, 55 (1999).

[25] T. Hyodo, A. Hosaka, E. Oset, A. Ramos and M. J. Vicente Vacas, Phys. Rev. C 68, 065203 (2003).

[26] L. S. Geng and E. Oset, Eur. Phys. J. A 34, 405 (2007).

[27] V. Bernard, N. Kaiser and U. G. Meissner, Int. J. Mod. Phys. E 4, 193 (1995).

[28] E. Oset, A. Ramos and C. Bennhold, Phys. Lett. B 527, 99 (2002) [Erratum-ibid. B 530, $260(2002)]$

[29] T. Hyodo and D. Jido, Prog. Part. Nucl. Phys. 67, 55 (2012).

[30] L. Roca and E. Oset, Phys. Rev. C 87, no. 5, 055201 (2013).

[31] L. Roca and E. Oset, Phys. Rev. C 88, no. 5, 055206 (2013).

[32] Z. -H. Guo and J. A. Oller, Phys. Rev. C 87 (2013) 035202.

[33] Y. Ikeda, T. Hyodo and W. Weise, Phys. Lett. B 706, 63 (2011).

[34] Y. Ikeda, T. Hyodo and W. Weise, Nucl. Phys. A 881, 98 (2012).

[35] M. Bazzi, G. Beer, L. Bombelli, A. M. Bragadireanu, M. Cargnelli, G. Corradi,

C. Curceanu (Petrascu) and A. d'Uffizi et al., Phys. Lett. B 704, 113 (2011).

[36] R. A. Schumacher and K. Moriya, arXiv:1303.0860 [nucl-ex].

[37] M. Mai and U. G. Meißner, arXiv:1411.7884 [hep-ph]. 